\title{
Reverse Engineering of Middleware for Verification of Robot Control Architectures*
}

\author{
Ali Khalili ${ }^{\dagger 1,2}$, Lorenzo Natale ${ }^{\ddagger 2}$ and Armando Tacchella ${ }^{\S 1}$ \\ ${ }^{1}$ DIBRIS, Università degli Studi di Genova, Via Opera Pia 13 - 16145 Genova - Italy \\ ${ }^{2}$ iCub Facility, Istituto Italiano di Tecnologia (IIT), Via Morego, 30 - 16163 Genova - Italy
}

\begin{abstract}
We consider the problem of automating the verification of distributed control software relying on publish-subscribe middleware. In this scenario, the main challenge is that software correctness depends intrinsically on correct usage of middleware components, but structured models of such components might not be available for analysis, e.g., because they are too large and complex to be described precisely in a cost-effective way. To overcome this problem, we propose to identify abstract models of middleware as finite-state automata, and then to perform verification on the combined middleware and control software models. Both steps are carried out in a computer-assisted way using state-of-the-art techniques in automata-based identification and verification. Our main contribution is to show that the combination of identification and verification is feasible and useful when considering typical issues that arise in the implementation of distributed control software.
\end{abstract}

\section{Introduction}

Publish-subscribe middleware such as ROS [15] and YARP [6] are becoming increasingly common in control architectures of modern robots. The main advantage of using middleware is that control modules can communicate seamlessly among each other and with device-specific APIs, possibly across different computing platforms. While operational scenarios for autonomous robots are becoming increasingly complex — see, e.g., the DARPA robotics

\footnotetext{
${ }^{*}$ The final publication is available at Springer via http://dx.doi.org/10.1007/ 978-3-319-11900-7_27

'Ali.Khalili@edu.unige.it

${ }^{\ddagger}$ Lorenzo.Natale@iit.it

§Armando.Tacchella@unige.it
} 
challenge [13] - the issue of dependability at all levels of a robot's architecture is getting more attention. In particular, if robots must be operated safely, control architectures must be verified against various requirements, which include also software specific properties, like deadlock or race avoidance. However, the task of verifying control software built on top of some middleware cannot be accomplished unless a precise model of the middleware is available, because a seemingly correct control code can easily lead the robot to unwanted states if middleware primitives are misused. An example of such case is when a sender assumes buffered communication to a receiver, but the channel is configured without buffering; if the sender expects acknowledgment for every message, but some message is lost, then a deadlock condition may ensue.

Insofar a component of a control architecture is assigned precise semantics, formal correctness verification is made possible, and many control software fallacies can be spotted at design time. However, developing a formal model can be difficult for large and complex middleware like ROS or YARP. A viable solution to this problem is to adopt automata-based identification techniques - see, e.g., [16] for a comprehensive list of references. The key idea is that the internal structure of a middleware component can be inferred by analyzing its interactions with an embedding context. Identification algorithms supply the component with suitable input test patterns to populate a "conjecture" automaton by observing the corresponding outputs; then, they check whether the conjecture is behaviorally equivalent to the actual component. When such an abstract model of the original component is obtained, it can be used as a stub to verify software components relying on it. This is where automata-based verification enters the scene. Given the inferred models of middleware components, and a model of the control software relying on them, Model Checking [14, 4] techniques provide an automated way to check behavioral properties about the composition of the models. In this way, confidence in a correct implementation of the overall control architecture is increased, and problems can be spotted before they cause expensive or even dangerous failures during robot's operation.

To demonstrate the effectiveness of our approach, we considered some relatively simple, yet significant, examples of control code built on top of YARP [6]. Our choice is dictated by several reasons, including a deep knowledge of the platform, and a fairly large installed base due to the adoption of YARP as the standard middleware of the humanoid iCub [12]. Moreover, YARP is a publish-subscribe architecture quite similar to other middleware widely used in the robotics community such as ROS. From the implementation point of view, YARP is a set of libraries written in $\mathrm{C}++$ consisting of more than $150 \mathrm{~K}$ lines of code. The purpose of YARP is to support modularity by abstracting algorithms and the interface to the hardware and operating systems. YARP abstractions are defined in terms of protocols. One of the main features of YARP is to support inter-process communication using 
a "port" abstraction. Our case studies focus mostly on the identification of various concrete mechanisms underlying this abstraction, e.g., buffered vs. non-buffered ports, and then to check control code relying on such implementations. Practical identification of different kinds of abstract models of YARP ports is enabled by our tool AIDE (Automata IDentification Engine) ${ }^{1}$. Model checking the composition of control code and middleware is accomplished with the state-of-the-art tool SPIN [8]. The results obtained combining AIDE and SPIN, albeit still preliminary, show that our approach is promising for the identification and verification of control-intensive parts of the code, i.e., those parts where the complexity of the code raises from control flow rather than data manipulation.

The remainder of this paper is organized as follows. In Section 2, a short summary of background and the related works will be provided. Section 3 introduces and motivates our YARP-based case studies. Section 4 presents our experiments on identification and verification. Finally, some concluding remarks and possible directions of future works are given in Section 5 .

\section{Background}

We define an interface automaton (IA) as a quintuple $P=\left(I, O, Q, q_{0}, \rightarrow\right)$ where $I$ is a set of input actions, $O$ is a set of output actions, $Q$ is a set of states, $q_{0} \in Q$ is the initial state of the system, $\rightarrow \subset Q \times(I \cup O) \times Q$ is the transition relation, and the sets $O, I$ and $Q$ are finite, non-empty and mutually disjoint. Our definition of IA is the same given in [1], which does not take into account the possibility of formalizing hidden actions. Since we wish to infer IAs as models of middleware components, we can neglect such actions without losing generality in our context. The set of all actions $A=I \cup O$ is the action signature of the automaton. Given a state $q \in Q$ and an action $a \in A$, we define the next state function $\delta: Q \rightarrow 2^{Q}$ as $\delta(q, a)=\left\{q^{\prime} \mid q \stackrel{a}{\rightarrow} q^{\prime}\right\}$, where we write $q \stackrel{a}{\rightarrow} q^{\prime}$ to denote that $\left(q, a, q^{\prime}\right) \in \rightarrow$. An action $a \in A$ is enabled in a state $q \in Q$ if there exists some $q^{\prime} \in Q$ such that $q \stackrel{a}{\rightarrow} q^{\prime}$, i.e., $|\delta(q, a)| \geq 1$. A state $q \in Q$ wherein all inputs are enabled is input-enabled, and so is an automaton wherein all states are input-enabled. An input-enabled IA is also known as I/O automaton [11. Given a state $q \in Q$, the set $\operatorname{out}(q) \subseteq Q$ of observable actions is the set of all actions $a \in O$ where $a$ is enabled in $q$. If $\operatorname{out}(q)=\emptyset$, then $q$ is called suspended or quiescent. According to [5], an execution fragment of the automaton is a finite alternating sequence of states and actions $u_{0}, a_{0}, u_{1}, \ldots, u_{n}$ such that $u_{i} \in Q, a_{i} \in A$ and $u_{i} \stackrel{a_{i}}{\rightarrow} u_{i+1}$ for all $0 \leq i<n$.

Automata-based Inference Automata-based identification (also, automata learning) can be divided into two wide categories, i.e., passive and active

\footnotetext{
${ }^{1} \mathrm{AIDE}$, developed in C\#, is an open-source software: http://aide.codeplex.com
} 
learning. In passive learning, there is no control over the observations received to learn the model. In active learning, the target system can be experimented with, and experimental results are collected to learn a model. Whenever applicable, active learning is to be preferred because it is computationally more efficient than passive learning - see [9] for details. Furthermore, active learning is not affected by a potential lack of relevant observations because it can always query for them. However, active learning requires that the target system is available for controlled experimentation, i.e., it cannot be performed while the target is executing. The basic abstraction in active learning as introduced by Angluin in [2], is the concept of Minimally Adequate Teacher (MAT). In our case, it is assumed that a MAT exists and it can answer two types of questions, namely output queries and equivalence queries. An output query amounts to ask the MAT about the output over a given input string, whereas equivalence queries amount to compare a conjecture about the abstract model of a system with the system itself. The result of equivalence queries is positive if the model and the system are behaviorally equivalent, and it is a counterexample in the symmetric difference of the relations computed by the two automata, otherwise. In practice, since the system is unknown, equivalence queries must be approximated by, e.g., model-based testing. Our tool AIDE is a collection of learning algorithms for several abstract models, including IAs. In particular, we use the Mealy machine inference algorithm $L_{M}^{+}$[16] together with the approach presented in [1] to identify IAs. This model of identification is particularly suited in contexts where the behavior of the system is jointly determined by its internal structure, and by the inputs received from the environment - also called tester.

Formal Verification Automata-based verification — see, e.g., [3] — encompasses a broad set of algorithms and related tools, whose purpose is to verify behavioral properties of systems represented as automata. In particular, we consider algorithms and tools for Model Checking [14, 4]. The basic idea behind automata-based verification technique is to exhaustively and automatically check whether a given system model meets a given specification. In this approach, a property is specified usually in terms of some temporal logic, and the system is given as some kind of automaton. In this work, we use SPIN [8], a generic verification system that supports design and verification of asynchronous process systems. In SPIN, the models are specified in a language called PROMELA (PROcess MEta LAnguage), and correctness claims can be specified in the syntax of standard linear temporal logic (LTL). Several optimization techniques, including partial order reduction, state compression and bit-state hashing are developed to improve performance of verification in SPIN. Details on the encoding of IA into SPIN are given in Section 4. Here, we give a short overview on how verification 


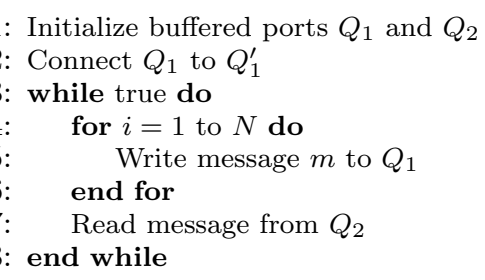

1: Initialize buffered ports $Q_{1}^{\prime}$ and $Q_{2}^{\prime}$

2: Set the reading mode of $Q_{1}^{\prime}$ as strict

3: Connect $Q_{2}^{\prime}$ to $Q_{2}$

4: while true do

5: $\quad$ for $i=1$ to $N$ do

6: $\quad$ Read message $m$ from $Q_{1}^{\prime}$

end for

8: $\quad$ Create a message and write it to $Q_{2}^{\prime}$

9: end while

Figure 1: Case study 1: An example code Planner (left) and Controller (right).

works in SPIN and similar tools. In SPIN, the global behavior of a concurrent system is obtained by computing an asynchronous interleaving product of automata, where each automaton corresponds to a single process. This means that, in principle, SPIN considers every possible interleaving of the atomic actions which every process is composed of. Technically, such product is often referred to as the state space or reachability graph of the system. To perform verification, SPIN considers claims specified as temporal formulas. Typical claims include, e.g., safety claims like "some property is always/never true", or liveness claims like "every request will be acknowledged". Claims are converted into Büchi automata, a kind of finite state automata whose acceptance condition is suitable also for infinite words. The (synchronous) product of automata claims and the automaton representing the global state space is again a Büchi automaton. If the language accepted by this automaton is empty, this means that the original claim is not satisfied for the given system. In other case, it contains precisely those behaviors which satisfy the original formula. Actually, the reachability graph is not computed up front because, if $n$ is the number of state variables, the computation would be exponential in $n$. Rather, the composition of the two automata is performed "on the fly", starting from the initial set of states of the system, and then considering the reachable ones given the process descriptions and the potential interleaving. SPIN terminates either by proving that some (undesirable) behavior is impossible or by providing a counterexample match.

\section{Case Studies}

Our motivation for this work is to to enable verification techniques for robot control software which uses middleware modules. In this section, we introduce two case studies. We focus on variations of the well-known producerconsumer paradigm. The reason is that similar situations are commonly found in robotic applications where loosely coupled modules are interconnected through publish-subscribe middleware and run concurrently. 
1: $\mathbf{P 1}$

2: Initialize buffered ports $Q_{1}$

3: Connect $Q_{1}$ to $Q_{1}^{\prime}$

4: for $i=1$ to $N_{1}$ do $1: \mathbf{P 3}$

5: //Do the job $\quad 2:$ Initialize buffered ports $Q_{1}^{\prime}$ and $Q_{2}^{\prime}$

6: $\quad$ Send message $m$ to $Q_{1} \quad$ Set the reading mode of $Q_{1}^{\prime}$ and $Q_{2}^{\prime}$ as strict

7: end for

1: P2

2: Initialize buffered ports $Q_{2}$

4: for $i=1$ to $N_{3}$ do

5: $\quad$ Read message $m_{1}$ to $Q^{\prime}$

3: Connect $Q_{2}$ to $Q_{2}^{\prime}$

4: for $i=1$ to $N_{2}$ do

$/ /$ Do the job

Send message $m$ to $Q_{2}$

7: end for

Figure 2: Case study 2: An example of two producers $\left(P_{1}\right.$ and $\left.P_{2}\right)$ and one consumer $\left(P_{3}\right)$ which are using YARP buffered port for their communication.

Case Study 1. We consider two software components that exchange messages with loose synchronization. A practical example is a Planner (P1) that generates a set of $N$ via points for a Controller $(P 2)$. The latter takes responsibility to execute each requests, in a variable amount of time. The Planner does not wait for execution of the individual commands but rather sends all $N$ messages to the Controller and then waits for a synchronization packet that signals the termination of the whole sequence. In a publish-subscribe architecture this can be achieved using two channels. The Planner uses the first channel (between $Q_{1}$ and $Q_{1}^{\prime}$ ) to send via points to the Controller, then waits for a message that acknowledges execution of the sequence from the Controller through the second channel (between $Q_{2}$ and $\left.Q_{2}^{\prime}\right)$. Since there is no synchronization, the buffering policy in the connections can affect the correct behavior of the system. In this application, the programmer assumes that connections are configured to queue at least $N$ messages. This may or may be not true in YARP where, by default, connections are configured to drop messages to reduce communication latencies ${ }^{2}$ The programmer therefore must override the default configuration of the connections to ensure that messages are queued and never dropped. Pseudo code for this scenario is given in Figure 1. Given this code, we can see that, if messages are dropped in the connections, a deadlock occurs. In practice, the robot may not only fail to follow the desired trajectory, but due to interpolation in the Controller, it may even end up in unsafe configurations.

Case Study 2. In publish-subscribe architectures, sensory information and commands travel on distinct channels. It is therefore common for components to receive information from multiple sources and synchronize their activities on data received from such connections. In this scenario, the consumer receives data from two producers. A practical example is a grasping

\footnotetext{
${ }^{2}$ This policy may seem counter-intuitive but it is fundamental for closed-loop control
} 


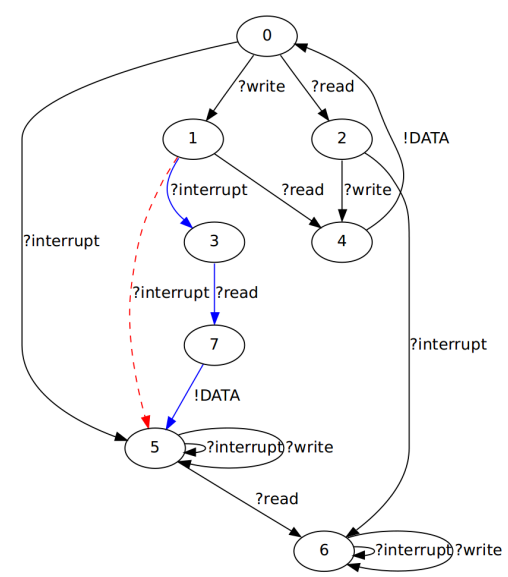

Figure 3: The identified model of a port with one reader, one writer, and one thread which interrupts the write port. The expected model would feature the dotted transition - from state 1 into state 5 - but the actual model identified by AIDE and implemented in YARP has the solid ones instead. Transitions labeled with "?" and "!" represent input and output actions, respectively.

application. Here the Tracker $(P 1)$ identifies the 3D position of the object in the work space (for example using stereo vision in the form of $(x, y, z)$ ). This information reaches the Controller $(P 2)$ through the connection between $Q_{1}$ and $Q_{1}^{\prime}$ which in turn computes the torque commands to the motors. Another component (P3) reads sensory data from a Force/Torque sensor placed in the kinematic chain, and publishes it on a separate channel. The Controller relies on this information to detect collisions and control the force exerted at the end-effector (connection between $Q_{2}$ and $Q_{2}^{\prime}$ ). The programmer of the Controller must decide how to read data from both connections. The crucial point is that these connections can become inactive. These might happen when no valid target is detected by the Tracker, or in situation where the Tracker was closed by the user or died unexpectedly. By default, YARP defines that readers wait for data on a port (blocking behavior). This allows tight synchronization and reduces latencies. An inexperienced programmer may read data from both channels using the default mode introducing an unexpected deadlock when Tracker does not produce data. The pseudo-code of this scenario is presented in Figure 2, The connection between $P 1$ and $P 2$ to $P 3$ is implemented using buffered ports with strict mode. To simulate the behavior where one of the producers stops sending data we added a counter $N_{i}$ to the main loop of each process. We verify the effects of different relative values of $N_{i}$ on the overall behavior.

In both the case studies describe above, we consider a combination of 
identification and verification to be detailed in Section 4. However, we would like to point out that identification alone is often useful to strengthen middleware, by helping the discovery of corner bugs that are elusive in common usage patterns. Out of many identification experiments that we conducted with YARP in our preliminary work, we show in Figure 3 the result of one which turned out as a report in the YARP bug-tracking system. In this example, we consider applying an interrupt method on a port. Interrupting a port is supposed to unblock any blocked thread waiting for the port. The model in Figure 3 is the one identified by AIDE for one port reader, one port writer, and a thread which interrupts the writing port. The model shows that interrupting a write port has been implemented so that it unblocks future writes, but it waits for completion of the current one, which was not the expected behavior. Indeed, this specific behavior was not documented, and never occurred in YARP practical applications, so it went unnoticed so far.

\section{Experiments}

Considering YARP port components as the system under learning (SUL) to be modeled as IA, we use AIDE to identify abstract models with different parameters. The configuration of components in the inference procedure is presented in Figure 4. As we mentioned in Section 2, the basic inference algorithm is $L_{M}^{+}$[16], and it is implemented in the module "MM Learning Algorithm" where "MM" stands for "Mealy Machine". The algorithm relies on a software component, called "MM Oracle" in Figure 4, whose task is to approximate the behavior of a MAT on a real system. Together, these two modules are the core of a Mealy machine inference program — "MM Learner" in Figure 4. Since we wish to identify YARP models as IAs, we connect a further component - "IA Translator" in Figure 4 - which implements the approach presented in [1] to identify IAs on top of a Mealy machine learning algorithm. All these modules are part of AIDE, and they collectively perform the task of "IA learner". The "System Wrapper" component (in $\mathrm{C}++$ ) bridges between the abstract alphabet on the learner side, and the concrete alphabet of the SUL. It manages different threads, handles the method calls in each thread, and the abstraction of messages - "bottles" in YARP terminology. To connect the wrapper to AIDE, we built a "Wrapper Proxy" which uses TCP/IP connections to facilitate identifying systems remotely, possibly across different computing architectures.

\subsection{Identification of Ports in YARP}

Configuration of Ports Considering a port connection, we examine different parameters which affect its behavior. In the case of a (standard) 


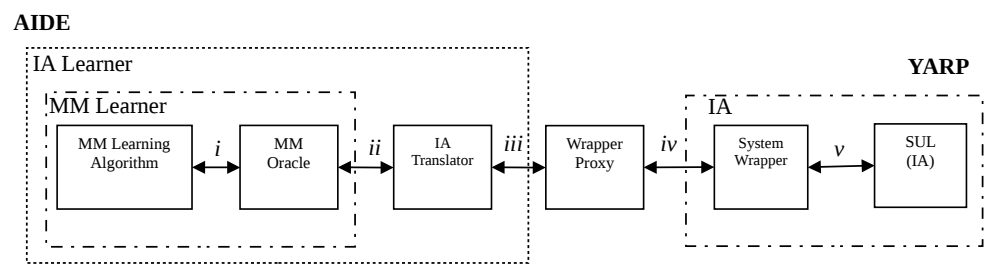

Figure 4: Components of the learning procedure. The connections are (i) queries asked to the MAT (MM Oracle), (ii) bi-directional translation of interface automata and Mealy machines, (iii) actions and events of the system, (iv) the TCP connection to remote system-wrapper, $(v)$ the abstraction/concretization made by the system wrapper.

YARP port in a scenario with one sender and one receiver, the type of communication is of a "send/reply" type, wherein the sender and the receiver are tightly coupled. In the case of buffered ports, the sender and the receiver enjoy more decoupling, in the sense that YARP takes care of the lifetime of the objects being transmitted through the port and it makes a pool of them, growing upon need. By default, a buffered port keeps the most recent message only. Therefore, messages that come in between two successive calls to read, might be dropped. If the so called "strict" mode is enabled, YARP will keep all received messages - like a FIFO buffer. Notice that, in this mode, the state space of the abstract automaton would be infinite. Therefore, to learn this model with AIDE, we limit the system to send no more than $N$ packets, i.e., we assume that the buffer will not exceed the maximum size of $N$ messages. In addition to a standard "Read" method which exists in normal ports, a non-blocking read feature is also available in buffered ports. Identification results for ports in various configurations are presented in Table 1 (top) ${ }^{3}$. We have also extended the alphabet of buffered ports to include non-blocking reading from the port for both strict and non-strict mode of reading - these two experiments are presented with an "** in Table 1. The reported measures include number of states $|Q|$ and transitions $|T|$ of the identified model, number of output ("\#MM") and equivalence ("\#EQ") queries in the learning algorithm, number of experiments on the SUL, and total time spent on learning. The behavior of a normal port is similar to a non-strict buffered port. In normal ports, both reading from port and writing to it are blocking, whereas in non-strict buffered port, writing is not a blocking primitive: if the buffer is not empty, the second write will overwrite the previous message. In buffered ports with strict mode enabled, writing to the port is non-blocking, but the difference compared to non-strict mode is

\footnotetext{
${ }^{3}$ All the experiments in this Section have been carried out on a Sony Vaio laptop with Core2Duo 2.26GHz CPU and 4GB of RAM on Ubuntu 12.04.
} 
Table 1: The result of inference for different models in YARP port component (top), and inference for different maximum size of buffer $(N)$ in YARP buffered port (bottom). In the topmost table, for buffered ports, we consider $N=3$.

\begin{tabular}{|l|c|c|c|c|c|r|}
\hline \multicolumn{1}{|c|}{ Model } & $|Q|$ & $|T|$ & \#MM & \#EQ & \#Experiments & Time $(\mathrm{s} \times 1000)$ \\
\hline \hline port & 4 & 5 & 18 & 1 & 33 & 0.6 \\
\hline buffered port (non-strict) & 4 & 6 & 35 & 2 & 50 & 1.0 \\
\hline buffered port (strict) & 8 & 11 & 132 & 4 & 180 & 2.0 \\
\hline buffered port (non-strict) & 4 & 8 & 63 & 2 & 114 & 2.5 \\
\hline buffered port (strict)* & 8 & 16 & 224 & 4 & 323 & 12.0 \\
\hline
\end{tabular}

\begin{tabular}{|c|c|c|c|c|c|r|}
\hline Size & $|Q|$ & $|T|$ & \#MM & \#EQ & \#Experiments & Time $(\mathrm{s} \times 1000)$ \\
\hline \hline 1 & 4 & 5 & 18 & 1 & 32 & 1.0 \\
\hline 2 & 6 & 8 & 54 & 3 & 86 & 2.1 \\
\hline 3 & 8 & 11 & 132 & 4 & 180 & 2.0 \\
\hline 4 & 10 & 14 & 225 & 6 & 260 & 10.0 \\
\hline 5 & 12 & 17 & 504 & 7 & 396 & 17.0 \\
\hline 6 & 14 & 20 & 987 & 8 & 531 & 31.0 \\
\hline
\end{tabular}

that the buffer does not drop older packets and it acts as a first-in-first-out buffer. In this case, the state space of the model would be infinite, and thus the buffer should be limited to a maximum size for finite state identification to work. These behavioral differences account for the different number of states and transitions in the identified models, as reported in Table 1. We have also considered the effects of increasing the maximum buffer size $(N)$ in buffered ports when the reading mode is set to be strict. These results are shown in Table 1 (bottom). For $N=1$, the observed model is the same as normal ports, and by increasing the size, the size of the model grows gradually. Notice that, if $e(N)$ denotes the number of experiments as a function of buffer size $N$, then we see that $e(N)$ grows more than proportionally with $n$. The CPU time spent for identification grows even faster due to some overheads in our current implementation. In particular, the capability of resetting the SUL is required by the identification algorithm. In our case, resetting the SUL includes releasing all the resources of the system, i.e., ports and threads working with them, and initializing the system again. This operation is performend in the system wrapper, and, in all our experiments, more than $95 \%$ of the total identification time is spent to reset the SUL. We expect that working on this bottleneck should enable us to experiment with larger buffer sizes, and also to infer a more accurate growth estimate for $e(n)$. Another issue which might affect the efficiency is the TCP/IP connection through the Wrapper Proxy, whereas calling YARP functions directly in AIDE would slightly decrease the identification time. 
Technical remarks Since the inferred models are deterministic, identical queries should produce the same answer. Therefore, we can cache the queries to avoid expensive repetitions. This is done by storing a tree of execution traces which can be exploited to avoid an experiment on a system whenever the corresponding query is a prefix of another one which has been already executed. In our implementation, the cache query is used as a filter between the MAT and the SUL, which makes it transparent from the MAT's point of view. Our reports above include only the actual number of queries on the system. Furthermore, in all of our experiments, less than $0.5 \%$ of the identification time was spent in the learning algorithm. The most time-consuming parts are network communication, system reset, and thread management. As we have mentioned above, one reason for such inefficiencies is that the wrapper uses several delays to make sure it is obtaining the correct result, since obtaining even one wrong observation in the output or equivalence queries would result in failing to learn a correct model.

\subsection{Verification}

The conversion of IAs inferred by AIDE into a PROMELA model is accomplished as follows. Every model is translated into one process type which communicates with two unbuffered channels - to simulate synchronous communication. These are InChannel and OutChannel, and their task is to receive input actions from environment, and emit output events correspondingly. To make the composition flexible, the input and output channels are the parameters of the process type. At any time, the next state is determined by the received input action (or the emitted event) and all the transitions are performed as atomic actions. In addition to PROMELA, AIDE is able to export inferred automata in DOT graph format, $\mathrm{C}++$ and the input language of other model checkers. The model of programs which use the ports are translated into automata as well. Here, the translation is manual, but in principle, it could be done in an automated fashion. Finally, the composition of the inferred model with the code model is done automatically by SPIN.

Case study 1 The results of verification, including the number of generated states by the model checker, the consumed memory, time and result of verification, are reported in Table 2 (top). Considering the identified model of a buffered port with non-strict mode of reading, SPIN finds a deadlock in the model after exploring 39 states, although the whole state space has about $16 \mathrm{~K}$ states. In fact the problem arises if the client sends packets too quickly through a YARP port configured for non-strict mode. In this case, there is a concrete chance that the server misses some of the packets, and a deadlock occurs. For strict mode, we consider a specific size of buffer $N$, namely $N=1$ and $N=6$. 
Table 2: Results of SPIN for the first case study (top) and second case study (bottom). In the topmost table, for buffered port, non-strict mode of reading and strict mode of reading for $(N=1$ and $N=6)$. In the bottommost table, different $N_{i}$ 's and size of buffer. The last row is the result of model checking with non-blocking read from $Q_{1}^{\prime}$

\begin{tabular}{|c|c|c|c|c|}
\hline Model & \#States & Memory(MB) & Time(s) & Conclusion \\
\hline \hline Buffered Port (non-strict) & 38 & 128 & 0.01 & deadlock \\
\hline Buffered Port (strict, $N=1$ ) & $15 \mathrm{~K}$ & 129 & 0.04 & OK \\
\hline Buffered Port (strict, $N=6)$ & $42 \mathrm{~K}$ & 132 & 0.09 & OK \\
\hline
\end{tabular}

\begin{tabular}{|c|c|c|c|c|r|c|c|}
\hline$N_{1}$ & $N_{2}$ & $N_{3}$ & Size & \#States & Time(s) & Memory(MB) & Conclusion \\
\hline \hline 100 & 100 & 100 & 1 & $8 \mathrm{~K}$ & 0.02 & 129 & OK \\
\hline 90 & 100 & 100 & 1 & 790 & 0.01 & 128 & deadlock \\
\hline 100 & 100 & 100 & 6 & $128 \mathrm{~K}$ & 0.33 & 140 & OK \\
\hline 90 & 100 & 100 & 6 & 1930 & 0.02 & 128 & deadlock \\
\hline 200 & 200 & 200 & 6 & $519 \mathrm{~K}$ & 3.29 & 176 & OK \\
\hline 180 & 200 & 200 & 6 & 3820 & 0.04 & 129 & deadlock \\
\hline $90 *$ & 100 & 100 & 6 & $19 \mathrm{M}$ & 91.00 & 1300 & OK \\
\hline
\end{tabular}

Case study 2 As before, we perform verification for buffered port with strict mode of reading. The results are presented in Table 2 (bottom). We examined different values for $N_{1}, N_{2}$ and $N_{3}$ and the size of buffer. As shown in the Table, when $N 1=N 2=N 3$ all processes finish successfully. But if either $N 1$ or $N 2$ are less than $N 3, P_{3}$ will be stuck as expected. Indeed, in situations where $P_{1}$ may finish sooner than $P_{3}$, the solution would be to change reading from $Q_{1}^{\prime}$ (line 5 in Figure 2 to a non-blocking read. Using the corresponding model and the maximum buffer size of 6 , SPIN can prove the non-existence of deadlock in $91 \mathrm{CPU}$ seconds - last row of Table 2 (bottom).

\section{Conclusion}

In this paper, we show how to exploit automata-based inference and verification techniques to identify port components of YARP middleware, and to verify control software build on top of them. Since YARP is the middleware of choice in the humanoid iCub, AIDE can enable the adoption of precise techniques for testing and verification of relevant components in iCub's control architecture.

To the best of our knowledge, this is the first time that a combination of identification and verification techniques is applied successfully in robotics. Similar contributions appeared in a series of works by Doron Peled and others - see, e.g., 7] for the most recent work - with hardware verification as the main target. However, our approach is more general since it decouples identification techniques from verification techniques, and it enables the 
combination of different flavors of such techniques.

Considering the current limitations of our work, we see (non)determinism and scalability as the two main issues. As for nondeterminism, it is well known that middleware can respond in different ways according to external events which are never completely under control. The algorithms that we have considered here assume that the middleware is behaving deterministically, which might turn out to be an unrealistic assumption. However, in a recent contribution [10], we have shown how to deal with nondeterminism when learning Mealy machines, and we expect to be able to extend this result also to IAs. Scaling to more complex components is a challenge for our future research agenda. In spite of harsh computational-complexity results, both identification and verification tools have a record of success stories in dealing with industrial-sized systems. Furthermore, AIDE already enables developers to check their code against common errors such as, e.g., incorrect port flagging, and it has also been useful in supplying YARP creators with corner bugs that helped them to improve some basic functionality of platform. We expect that improving the bottlenecks due to resetting the system, i.e., managing ports and related threads in the system wrapper, will improve the capacity of our techniques.

\section{References}

[1] Aarts, F., Vaandrager, F.: Learning I/O automata. CONCUR 2010Concurrency Theory pp. 71-85 (2010)

[2] Angluin, D.: Learning regular sets from queries and counterexamples. Information and computation 75(2), 87-106 (1987)

[3] Baier, C., Katoen, J.: Principles of model checking. MIT press Cambridge (2008)

[4] Clarke, E., Emerson, E., Sistla, A.: Automatic verification of finitestate concurrent systems using temporal logic specifications. ACM Transactions on Programming Languages and Systems (TOPLAS) 8(2), 263 (1986)

[5] De Alfaro, L., Henzinger, T.: Interface automata. ACM SIGSOFT Software Engineering Notes 26(5), 109-120 (2001)

[6] Fitzpatrick, P., Metta, G., Natale, L.: Towards long-lived robot genes. Robotics and Autonomous systems 56(1), 29-45 (2008)

[7] Groce, A., Peled, D., Yannakakis, M.: Adaptive model checking. Logic Journal of IGPL 14(5), 729-744 (2006) 
[8] Holzmann, G.J.: The SPIN model checker: Primer and reference manual, vol. 1003. Addison-Wesley Reading (2004)

[9] Kearns, M., Vazirani, U.: An introduction to computational learning theory. MIT press (1994)

[10] Khalili, A., Tacchella, A.: Learning nondeterministic mealy machines. In: Proceedings of the $12^{\text {th }}$ International Conference on Grammatical Inference (ICGI) (2014), to appear

[11] Lynch, N.A., Tuttle, M.R.: Hierarchical correctness proofs for distributed algorithms. In: Proceedings of the sixth annual ACM Symposium on Principles of distributed computing. pp. 137-151. ACM (1987)

[12] Metta, G., Natale, L., Nori, F., Sandini, G., Vernon, D., Fadiga, L., von Hofsten, C., Rosander, K., Lopes, M., Santos-Victor, J., et al.: The iCub Humanoid Robot: An Open-Systems Platform for Research in Cognitive Development. Neural networks: the official journal of the International Neural Network Society (2010)

[13] Pratt, G., Manzo, J.: The DARPA Robotics Challenge [Competitions]. Robotics \& Automation Magazine, IEEE 20(2), 10-12 (2013)

[14] Queille, J., Sifakis, J.: Specification and verification of concurrent systems in CESAR. In: International Symposium on Programming. pp. 337-351. Springer (1982)

[15] Quigley, M., Conley, K., Gerkey, B., Faust, J., Foote, T., Leibs, J., Wheeler, R., Ng, A.Y.: ROS: an open-source Robot Operating System. In: ICRA workshop on open source software. vol. 3 (2009)

[16] Shahbaz, M.: Reverse Engineering Enhanced State Models of Black Box Software Components to Support Integration Testing. Ph.D. thesis, Institut Polytechnique de Grenoble, Grenoble, France (2008) 